\title{
Location Privacy in the Era of 5G
}

\author{
Alexander Schacht, Kayla Foster, and John Roth \\ Electrical and Computer Engineering Department \\ Naval Postgraduate School \\ Monterey, United States of America \\ alexander.schacht@nps.edu kayla.foster@nps.edu jdroth@nps.edu
}

\begin{abstract}
Fifth Generation (5G) wireless technology is ushering in a new age of interconnectivity, and as it does definitions of privacy may well change with it. In this paper we will focus on the changing nature of location privacy, while aspiring to increase community cognizance of vulnerabilities within the $5 \mathrm{G}$ network that threaten end-user privacy. To this end, we will address the statistical efficacy of a multilateration attack utilizing timing advance commands within the $5 \mathrm{G}$ cloud radio access network, by showcasing that such an attack meets the CramérRao Lower Bound (CRLB) across each subcarrier spacing. We will also demonstrate how position estimates within 5G can be further refined using methods previously shown to be effective in Long-Term Evolution (LTE) Networks. Lastly, we will demonstrate the attack in a simulated environment modeled after the conference grounds, using current real-world deployments of $5 \mathrm{G}$ on Kauai.
\end{abstract}

\section{Introduction}

Privacy is a fundamental part of any society. It is inextricably woven into the fabric of our everyday lives, to the point of expectation, which when not met, is often seen as an egregious violation of a most basic right. That being the case, we must ask ourselves, how is privacy defined? To date, there have been numerous definitions of privacy, with societies and in fact individuals, often reevaluating what privacy means to them. In one of the first significant and influential works on consumer data privacy and data protection [1], Professor Alan Westin, asserts this definition:

Privacy is the claim of individuals, groups, or institutions to determine for themselves when, how, and to what extent information about them is communicated to others.

The above is readily applied to location privacy and sets a framework for the discussion of why it is changing, how it is threatened, and inspires the most important question, one which we will answer in due time; "Who cares?"

\subsection{The Capricious Nature of Privacy}

Each individual's perspective on what should remain private is influenced by their own internal beliefs, as well as extrinsic pressure from the societal norm, which shifts with time and location. What today seems normal to share about yourself, may have been, or more likely was indeed, entirely anathema 100 years ago.

1.1.1. The Why. The digital age has brought on an entirely unprecedented and almost dogmatic change in when, how, and what we share about ourselves. Social media, location based services (LBS), targeted advertisements, data/internet usage tracking, have all whittled away at the idealization of "private-life" vs "public-life" that the two seem interchangeable. This will only become more blurred as the Internet of Things (IoT) comes into full swing with multibillion increases in number of connected devices per year (see Figure 1), that with the promise of $5 \mathrm{G}$, device-to-device (D2D) communication, and access points on every corner, will be streaming a near constant supply of information about end users.

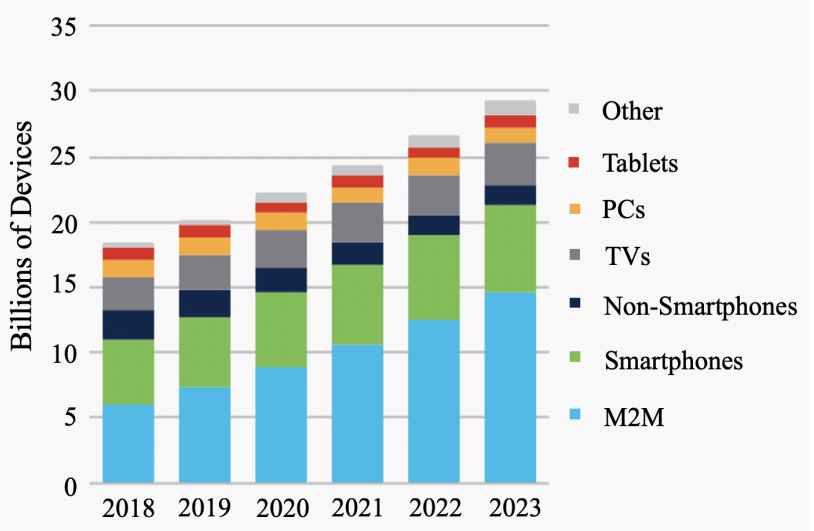

Figure 1: Projection of number of connected devices through 2023 [2]. 
1.1.2. The How. For location privacy, this is especially true. As the prevalence and precision of location-aware devices increases, an end user's privacy concerning their location decreases. This may seem an almost trivial statement, as there has been a long-held generalization that the utility provided by LBS, across the realms of safety (enhanced911 (E-911) calls), commerce (store advertisements based on location), fitness (distance/route tracking), transportation (route selection/habit tracking), etc, outweighs this loss in privacy. However, we will show that by the nature of the $5 \mathrm{G}$ protocol and network architecture, end user location data is at risk to anyone, with a relatively small amount of technical acumen and resources at their disposal.

\subsection{Previous Work}

Localization of user equipment (UE) as a requirement was first codified in the 1996 Federal Communications Commission (FCC) mandate for standardized accuracy requirements for E-911 calls [3]. These requirements have been revised a multitude of times with accuracy requirements becoming more precise with each iteration, resulting in far reaching effects on the use of users' location data, and setting the pace for industry.

Rosado et al identify the five fundamental positioning techniques utilizing radio signals as trilateration, triangulation, proximity, scene-analysis, and hybrid [4]. We will focus entirely on trilateration, or more generally, multilateration. Multilateration is a technique where a UE position solution is calculated based on the intersection of common geometric constructs based on distance measurements between the UE and reference transmitter/receiver (time of arrival (ToA), received signal strength (RSS), angle of arrival (AoA), etc). Figure 2 displays this localization technique succinctly. To meet regulatory requirements, large sums of research have been conducted on mobile and network based trilateration techniques that have shown these techniques to be a valid method for achieving the aforementioned requirements [5].

\subsection{Novel contributions}

This paper will focus on addressing the statistical efficiency of a multilateration technique utilizing inherent timing management controls across the fresh numerologies present in the 5G protocol. We will also demonstrate the effectiveness of an enhancement algorithm to increase position fidelity and showcase a simulated attack on a conference attendee.

The remainder of this paper is organized as follows. In Section 2 we will cover an overview of the Cloud Radio Access Network (CRAN) architecture, sub-carrier spacing (SCS)s, and timing advance (TA) command. In Section 3 we discuss our proposed attack framework. In Section 4 we will discuss the CRLB, and show a comparative analysis of the CRLB across each SCS and position accuracy utilizing Cellular Synchronization Assisted Refinement (CeSAR). In Section 5 we exhibit our simulated implementation on
Kauai, following up with an exploration of mitigation techniques in Section 6. We present our closing remarks in Section 7.

\section{Foundations}

Here follows a baseline introduction to key concepts leveraged in this research.

\subsection{The Cloud Radio Access Network}

In order to meet the demanding aspirations of $5 \mathrm{G}$, it was determined that the classical network architecture and protocols must be overhauled, thus the CRAN architecture was designed. The varied divergences from the established design can be grouped into two predominant categories of physical restructuring of the network and interface updates.

The physical scheme of the network, detailed in Figure 3, is composed of a grouping of centrally located baseband unit (BBU)s, in what is known as a "BBU pool", and their associated remote radio head (RRH)s. Each pool is stationed in its servicing geographic region, and connected via a fronthaul network to numerous RRHs densely distributed close to UEs. For this study we assume a standard fiber deployment, though there have been other physical layer proposals [6]. This is in stark contrast to the traditional LTE framework, where the BBU and RRH would be deployed together as the singular base station.

Interface updates are required as the legacy common public radio interface (CPRI), an industry cooperation used in LTE [7], would necessitate a substantial increase in the capacity required to maintain the proposed data rates. It

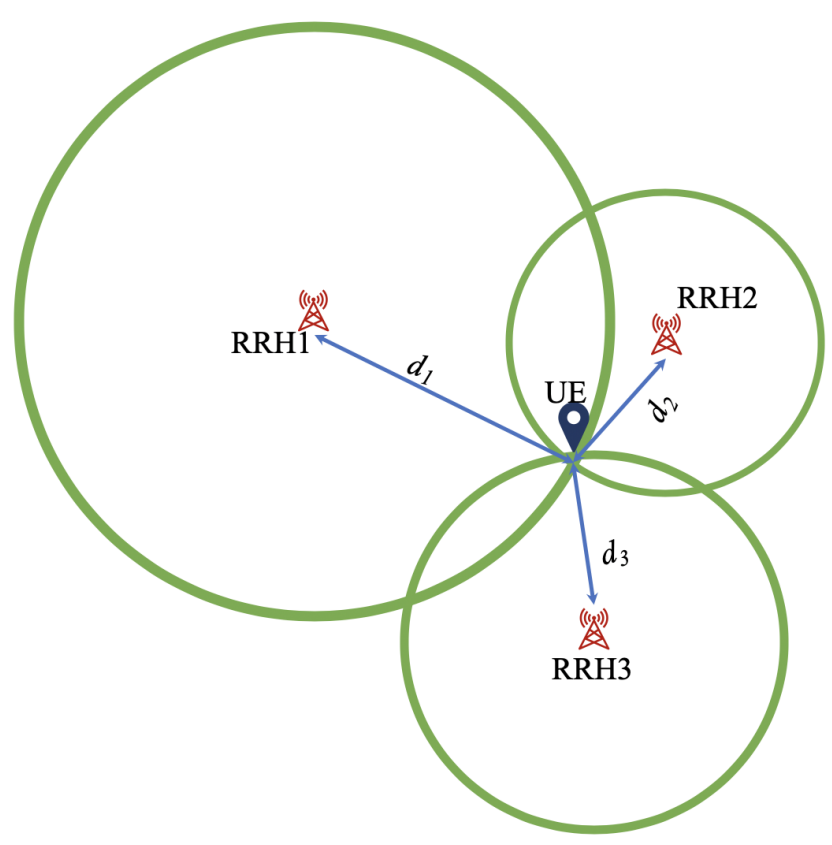

Figure 2: Example of trilateration in a wireless network. 


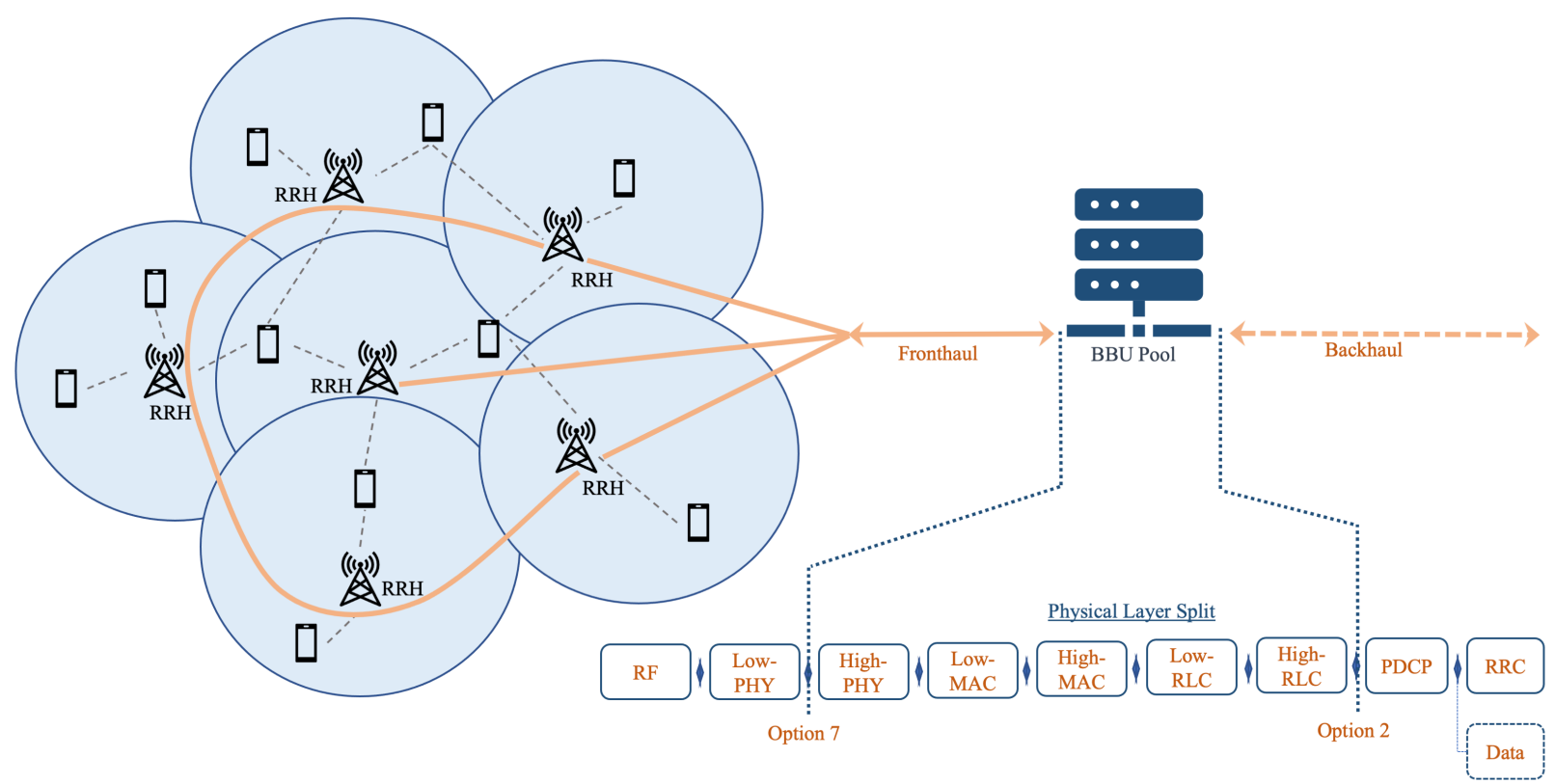

Figure 3: Example of C-RAN Architecture with physical layer splits identified.

would also eliminate the ability for network capacity to be scaled based on real-time traffic volume, as the capacity requirements would have to be fixed, reducing network efficiency, and missing a notable mark for 5G. New interfaces are being designed by the major industry leaders (Institute of Electrical and Electronics Engineers (IEEE), 3rd Generation Partnership Project (3GPP), 5G Infrastructure Public-Private Partnership (5GPP)) and others to address the above issues. The CPRI group's response is the enhanced CPRI (eCPRI). How these interfaces work to find a balance between latency, efficiency, and customizability is founded on the concept of functionally splitting the physical layer protocols. The physical layer split (PLS) architecture, as defined by 3GPP, [8] gives numerous options for network engineers to decide how they want to divvy out responsibilities between the BBU and RRH. For this paper we will assume that our target network is operating using eCPRI with an option $7 / 2$ fronthaul/backhaul, wherein the BBU maintains all radio link control and media access control layer functions, while lower physical layer functions, such as modulation and fast Fourier transforms are delegated to the RRH [9]. This results in the BBU conducting the majority of the digital baseband radio functions, while the RRH carries out mostly analog radio frequency tasks, which allows for the cost effective spatial separation desired [6].

\subsection{Numerologies}

To enable a highly flexible network, 5G New Radio (NR) established multiple numerologies $\mu$, defined by the specific
SCS [10]. The SCSs are defined as

$$
S C S=15 \times 2^{\mu} \mathrm{kHz} \text { for } \mu \in[0,4] .
$$

Therefore, supporting a range of 15 (standard LTE) to 240 $\mathrm{kHz}$, where higher SCS realizes the increased bandwidth and a shorter transmission time interval required for mmWave (carrier frequency bands above $24 \mathrm{GHz}$ ) and delay sensitive services, while lower is optimal for high physical layer throughput [11]. Other than the primary intended effects described above, the alteration of the SCS and Cyclic Prefix (CP) for each numerology provides an ancillary service in terms of localization via an exploitation of timing management in $5 \mathrm{G}$.

\subsection{NR Timing Advance Command}

The LTE TA command is made up of two notable parts, the timing advance value $N_{T A}$ and the Timing Advance Group (TAG) [12]. Its purpose is to ensure that as a UE moves within the serviceable environment, its transmissions arrive at the RRH during its given time slot. As the UE moves, the propagation time for its transmissions will change, significantly at times, requiring for some method of synchronization.

The TAG, is a 2-bit field that allows unique association of a TA command to a particular RRH (maximum of 4) specifically to account for communication with multiple RRHs, through carrier aggregation [13] and the high unlikelihood that they are equidistant from the UE. $N_{T A}$ has 


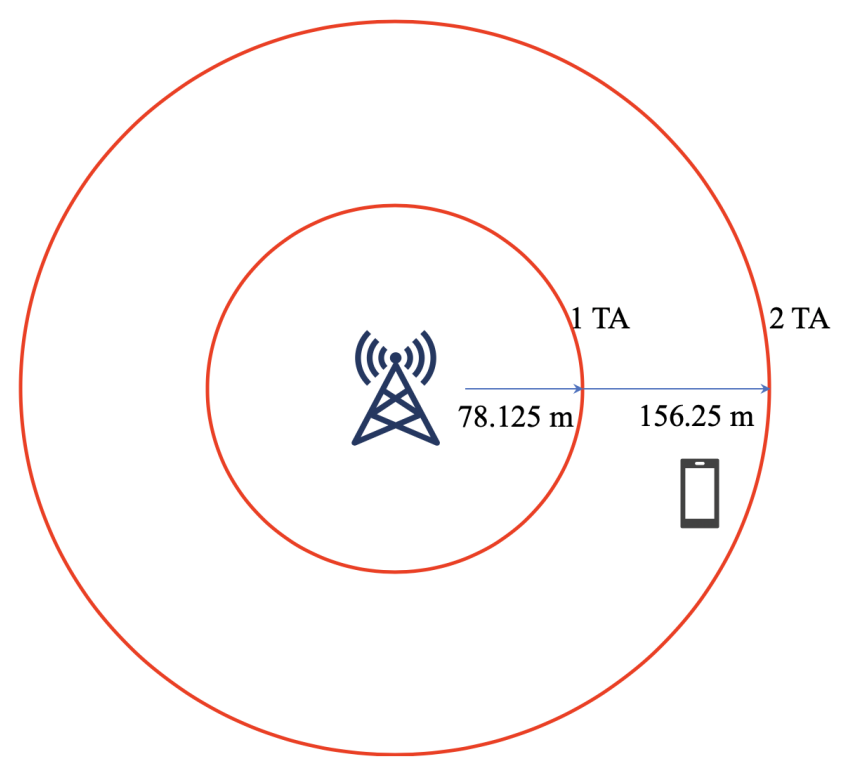

Figure 4: Visual representation of a TA command with TA $=2$ and the associated distances with $\mu=0$.

an associated fixed time value due to LTE's static basic time unit, $T_{s}$, detailed in equation (2).

$$
T_{s}=\frac{1}{\Delta f_{\text {ref }} \times N_{f, r e f}}=\frac{1}{15 \times 10^{3} \times 2048} \approx 32.6 \mathrm{nsec}
$$

where $\Delta f_{\text {ref }}$ is the LTE SCS and $N_{f, r e f}$ the maximum number of subcarriers. A singular TA value, represented as an integer, accounts for 16 slot times such that

$$
N_{T A}=16 T_{s} .
$$

Utilizing equations (2) and (3) the one-way distance resolution can be calculated as shown

$$
r=\frac{c N_{T A}}{2}=78.125 \text { meters. }
$$

In employing the NR numerologies a new base unit of time is employed in [14]

$$
T_{c}=\frac{1}{\Delta f_{\max } \times N_{f}}=\frac{1}{480 \times 10^{3} \times 4096} \approx .51 \mathrm{nsec}
$$

where $\Delta f_{\max }$ is the maximum SCS and $N_{f}$ the maximum number of subcarriers. The relationship between $T_{c}$ and $T_{s}$ is stated in [10] by introduction of $\kappa$ defined as

$$
\kappa=\frac{T_{s}}{T_{c}}=64 .
$$

Slot time in NR is redefined as

$$
T_{s}=\frac{1}{\Delta f_{\text {ref }} \times N_{f, r e f}}=\frac{1}{15 \times 10^{3} \times 2^{\mu} \times 2048}
$$

resulting in each incremental TA per novel numerology ${ }^{1}$ being equivalent to

$$
N_{T A}=\frac{16 \kappa T_{c}}{2^{\mu}}
$$

where, based on equation (6), $T_{c}$ is cancelled out leaving

$$
N_{T A}=\frac{16 T_{s}}{2^{\mu}} .
$$

Therefore, making use of equations (4) and (9), the new distance resolutions are calculated as

$$
r=\frac{c N_{T A}}{2}=\frac{78.125}{2^{\mu}} \text { meters }
$$

evincing the dependence of 5G TA distance resolutions on the associated numerology. Table 1 summarizes the new resolutions while Figure 4 grants a visual understanding of the TA.

TABLE 1: 5G NR Numerology Distance Resolutions

\begin{tabular}{ccc}
\hline \hline $\boldsymbol{\mu}$ & Distance Resolution (m) & Subcarrier Spacing (kHz) \\
\hline 0 & 78.125 & 15 \\
1 & 39.06 & 30 \\
2 & 19.53 & 60 \\
3 & 9.77 & 120 \\
4 & 4.88 & 240 \\
\hline
\end{tabular}

\subsection{Statistical Efficiency of an Estimator}

One of the main goals of this investigation, is to show that our method for finding a maximum likelihood estimate (MLE) utilizing TAs is statistically sound. To achieve this we consider a well-established bound for the minimum variance of a parameter estimate; the CRLB [15]. The bound is defined as

$$
\operatorname{Var}_{\theta}\{T\} \geq I^{-1}(\theta)
$$

where $T$ is an unbiased estimator of the parameter $\theta$ and $I(\theta)$ is the Fisher information with respect to $\theta$. The CRLB's applicability in LTE TA localization has been shown extensively in [16], where the unbiased estimator is the UE position MLE and the parameter to be estimated is the true position. The authors also show that for each TA distance resolution, $r$, the root mean square error (RMSE) is bounded above the CRLB for $r \lesssim 3.4 \sigma$, where $\sigma$ is a measure of variation within the position estimates. Our work extends this to $5 \mathrm{G} \mathrm{NR}$ and the novel numerologies.

\section{Attack Schema}

The fundamental structure of this attack is to localize a victim using their aggregated TA information via whatever prudent method of collection available and a MLE algorithm. In a traditional LTE network, an antagonist would

\footnotetext{
${ }^{1}$ If $\mu=0$, the result is the expected legacy LTE $T_{s}$.
} 
place themselves advantageously to collect the information over the open-air interface; however, the updates to the physical layer in NR (ultra-densification, tight beamforming, use of massive multiple-input multiple-output (MIMO), etc) have resulted in this being less than effective at the higher SCSs and bands. In an ultra-dense environment, determining which RRH is in communication with a target UE would be non-trivial, and if found, positioning a sensor to receive the UE uplink burst in mmWave communications would require the sensor and receiving RRH to be nearly colinear, resulting in little to no extra positioning information provided, greatly diminishing the motivation to use CeSAR enhancement.

The CRAN architecture and discussed PLS has simply shifted this vulnerability by maintaining the unencrypted nature of the TA and passing it from the RRH to the $\mathrm{BBU}$ along the fiber fronthaul. In fact, this may have made the vulnerability worse as it allows for the possibility of collection on the aggregated data as the target moves within the environment.

\subsection{CeSAR}

As an addition to our standard attack method we will implement the CeSAR method documented in [17], as a passive enhancement to our localization technique. Though this enhancement is ineffective in particular $5 \mathrm{G}$ use cases, as described above, it is still entirely valid in NR environments operating in sub $6 \mathrm{GHz}$ and LTE frequencies, as will be the case in less densely populated locations. It involves the placement of a secondary device to listen for the uplink burst from the target UE to the RRH, creating another known distance to further increase the localization fidelity. The algorithm, as defined in [17] is detailed in Table 2.

\section{Initial implementation}

\subsection{Comparative Analysis of Statistical Efficiency}

To begin, we established the wireless architecture by establishing the UE at $[0,0]$ and then randomly distributing 4 RRHs within a $1 \mathrm{~km} \mathrm{x} 1 \mathrm{~km}$ area $^{2}$. The true distances from the RRH to the UE were calculated and introduced to gaussian noise, forming our distance estimates, $\hat{d}_{i}$, which were then quantized into their appropriate TAs. Utilizing the known range values for each TA, we then found the target's position MLE, $\hat{\boldsymbol{p}}$, through the employment of the Non-Linear Least Squares (NLLS) method presented in [18] and [19]. This involves the minimization of $\boldsymbol{x}=[x, y]^{T}$ in the following

$$
\hat{\boldsymbol{p}}=\underset{x}{\arg \min } \sum_{i=1}^{N}\left[d_{i}-\left\|\boldsymbol{x}-\boldsymbol{x}_{\boldsymbol{i}}\right\|\right]^{2}
$$

where $\hat{\boldsymbol{p}}=[\hat{x}, \hat{y}]^{T}, d_{i}$ is the distance from each RRH to the center of its TA ring, $\boldsymbol{x}_{\boldsymbol{i}}=\left[x_{i}, y_{i}\right]^{T}$ are the positions of

\footnotetext{
${ }^{2}$ For clarity, the UE at $[0,0]$ is the center point of this square area.
}

each RRH, and $i$ is an integer ranging from 1 to the total number of RRHs. From the solution to (12), the squared distance error, error ${ }_{d}^{2}$, is calculated squaring the distance between $\hat{\boldsymbol{p}}$ and the true UE position $\boldsymbol{p}$. Now that we have established our positioning error for this trial, we find the CRLB. Based on (11), we state that our mean squared error (MSE) for the UE position estimate is bounded by

$$
\operatorname{Var}\{\hat{\boldsymbol{p}}\} \geq \boldsymbol{I}^{-1}(\boldsymbol{x})
$$

where $\boldsymbol{I}$, for the purpose of multilateration-based position estimates is given by [18]

$$
\boldsymbol{I}=\left[\begin{array}{cc}
\sum_{i=1}^{N} \frac{\left(x-x_{i}\right)^{2}}{\sigma_{i}^{2} d_{i}^{2}} & \sum_{i=1}^{N} \frac{\left(x-x_{i}\right)\left(y-y_{i}\right)}{\sigma_{i}^{2} d_{i}^{2}} \\
\sum_{i=1}^{N} \frac{\left(x-x_{i}\right)\left(y-y_{i}\right)}{\sigma_{i}^{2} d_{i}^{2}} & \sum_{i=1}^{N} \frac{\left(y-y_{i}\right)^{2}}{\sigma_{i}^{2} d_{i}^{2}}
\end{array}\right],
$$

and $\mathrm{CRLB}=\operatorname{tr}\left(\boldsymbol{I}^{-1}\right)$ Once found, we compute the difference between our square error and the CRLB normalized by the $\mathrm{CRLB}^{3}$ such that

$$
\operatorname{ediff}_{\mathrm{CRLB}}=\frac{\operatorname{error}_{d}^{2}-\mathrm{CRLB}}{\mathrm{CRLB}} .
$$

This procedure is then conducted one-hundred thousand times for each numerology across values of $\sigma$ ranging from 1 to 30 . Finally the average ediff ${ }_{\mathrm{CRLB}}$ is found for each value of $\sigma$.

\section{Results}

As seen in Figure 5, for each numerology the ediff ${ }_{\mathrm{CRLB}}$, provided in 15, approaches 0 as $\sigma$ increases. The vertical dotted lines show the expected approximate value of $\sigma$ where the MLE for each numerology meets the CRLB, based on the theoretical bounding of $r \lesssim 3.4 \sigma$, described in Section 2.4. This theory is shown to be well founded as each numerology performed as expected with $\mu=0: 2$ the closest to this theoretical point of bounding, and $\mu=3: 4$ having slightly greater values of $\sigma$ for which they converge with the CRLB. This gives a baseline for values of $\sigma$ that will produce the most statistically efficient location estimate based on the numerology chosen. Moving forward in this paper, the remainder of the multilateration simulations take this into account, using static values of noise variation, in order to show the most accurate position estimates possible.

\subsection{CeSAR Enhanced Localization}

This portion of our investigation is dedicated to determining the potential gains of the CeSAR algorithm when used in conjunction with the NR numerologies, as well as the effectiveness of localization in $\mathbb{R}^{3}$. To this end, we first establish our RRH and UE deployment by randomly distributing and selecting 4 RRHs and $1 \mathrm{UE}$

\footnotetext{
${ }^{3}$ As the CRLB changes each iteration based on the relative positions of RRHs to the UE, normalizing by the CRLB is necessary to compare across trials.
} 
TABLE 2: Cellular Synchronization Assisted Refinement [17]

\begin{tabular}{|c|c|c|}
\hline Step & Procedure & Description \\
\hline 1 & cesar $\left(\boldsymbol{p}_{R R H}, \boldsymbol{p}_{\text {sensor }}\right.$, target crnti $)$ & Passive enhancement procedure utilizing sensors, rrh's and target crnti. \\
\hline 3 & sensor $\leftarrow$ rrh downlink & Sensor listens for the PSS/SSS from serving rrh. \\
\hline 5 & repeat & Repeat steps 2-4 for each. rrh \\
\hline 6 & $x \leftarrow$ observed crnti & Sensor will continue to decode packets. \\
\hline 7 & until & Sensor continues to search for target crnti. \\
\hline 10 & $t \leftarrow$ ta estimate $\mathrm{tx}$ & The TA is used to estimate the target ue's uplink transmission time. \\
\hline 11 & $t^{\prime} \leftarrow$ observed & observe uplink burst timing information from one of the $\mathrm{N}$ serving rrhs. \\
\hline 12 & $\Delta t \leftarrow t^{\prime}-t$ & $\begin{array}{l}\text { Utilizes information from step } 10 \text { to measure the propagation delay from the ue to the } \\
\text { sensor. }\end{array}$ \\
\hline 13 & $\hat{d}^{\prime}=\Delta t \times c$ & Convert to a distance measurement. \\
\hline 14 & $\hat{\boldsymbol{d}} \leftarrow\left[\hat{d}, \ldots, \hat{d_{N}}, \hat{d}^{\prime}\right]^{T}$ & $\begin{array}{l}\text { Additional distance measurement is added to the distance measurement obtained from } \\
\text { the } \mathrm{N} \text { serving rrhs. }\end{array}$ \\
\hline
\end{tabular}

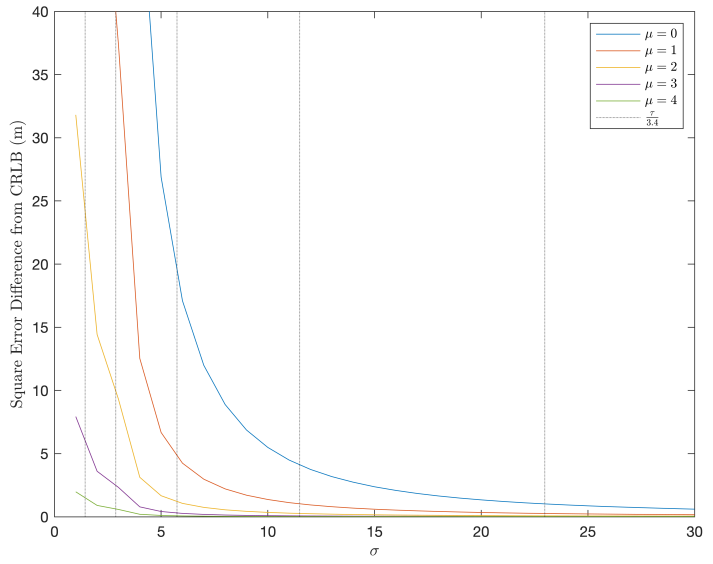

Figure 5: Difference between square error and CRLB for each SCS across $\sigma$ values.

each with randomly determined heights. Next, we follow a similar procedure to our standard implementation in the previous section for finding the MLE, particularly solving (12), without CeSAR enhancement, selecting $\sigma$ values consistent with meeting the CRLB for each numerology. Next, we enhance the previous findings by adding a singular CeSAR sensor. This is done in the same method established in Table 2. For simulation purposes, the CeSAR sensor is randomly placed in the environment, steps 1-13 are assumed to have been completed, and we utilize the true distance measurement between the sensor and UE. This distance is combined with the RRH measurements, and the enhanced MLE is calculated as before. The above procedure was then completed five-hundred thousand times for each numerology.

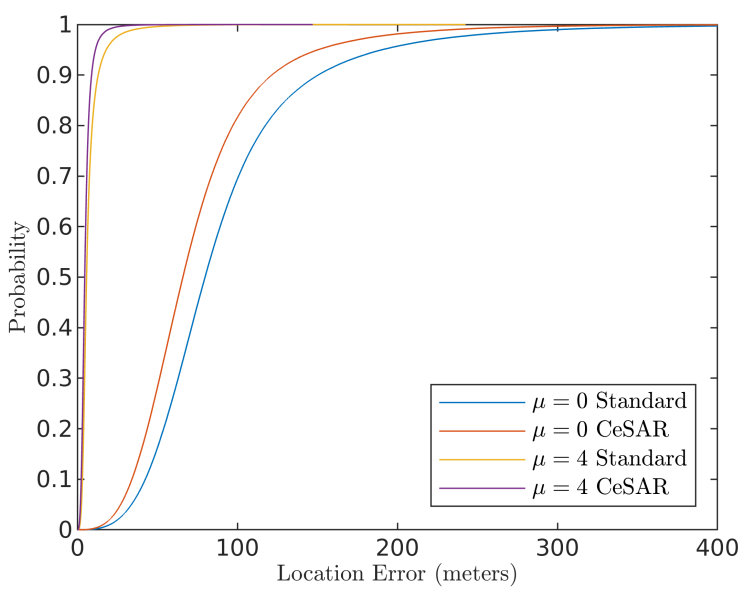

Figure 6: CDFs for both standard and CeSAR implementations for $\mu=0$ and $\mu=4$ respectively.

\section{Results}

The cumulative distribution function $(\mathrm{CDF}) \mathrm{s}$ for the lowest and highest SCSs are shown in Figure 6, with the results for a circular error probable (EP) of $90 \%$ and $95 \%$ for all SCSs are displayed in Table 3. $\mathrm{CEP}^{4}$ [20] relates the location distance error and confidence location error, by creating a ring of precision around a target, in which a chosen percentage of location estimates are contained $(90 \%$ and $95 \%$ respectively), where the radius is equal to the furthest location distance error among those contained points (see Figure 7)

A brief viewing of the above conveys the CeSAR algorithm's marked increase in positional accuracy for each numerology. A deeper look at Table 3, reveals that at its

${ }^{4}$ CEP 90\%: $\operatorname{Pr}\left[\|\hat{p}-p\|_{2}<C\right]=0.9$. 
worst, the algorithm increases position fidelity by $\approx 20 \%$ (see $\mu=0$ ) and $\approx 40 \%$ (see $\mu=4$ ) at its best, either of which are considered significant. These results support the use of CeSAR as an augmentation for TA-based localization attacks in $5 \mathrm{G} \mathrm{NR}$.

\section{Realization in Kauai}

The final portion of our paper presents a simulation of our localization attack on an unsuspecting HICSS attendee. This will be broken into two separate wireless architectures (displayed in Figures 7 and 8), the former being a real-world setup, the latter being a vision of the future ultra-dense (UD) $5 \mathrm{G}$ deployment. This split is based on the different use cases for the various numerologies. For the real-world type, the singular $\mathrm{RRH}$ is an operational $5 \mathrm{G}$ tower, the locations of the CeSAR sensors were chosen based on topography and angle from targets projected position and numerologies 0-2 will be utilized based on distance to the closest $\mathrm{RRH}^{5}$. The UD deployment is somewhat arbitrary, with an emphasis on attempting to have near constant line-of-sight conditions within the observable area to simulate a mmWave enabled UD system.

For increased realism, the UE, RRHs, and CeSAR sensors were first plotted in Google Earth to obtain global positioning system (GPS) coordinates. These positions were then converted to their values in meters. The real-world deployment was then run through the CeSAR enhanced algorithm to find the distance resolutions for $\mu=0$ through $\mu=2$, while the UD deployment is run through our standard algorithm. The reason for not running the latter through CeSAR enhancement is the low liklihood of being able to properly position a sensor in order to pick up the mmWave communications without already having a solid estimate of the target's position, due to mmWave communications' extremely small beam-width and controlled directionality.

Once position estimates in meters are obtained we convert back to latitude/longitude.

This procedure is repeated numerous times so that an average of the returned GPS coordinates can be taken to find our MLE of the target's position. For our procedure we ran the simulation ten times, one-hundred times, and one thousand times, and found that, though increased number of runs also increases the position estimate accuracy, it does so minimally. This coupled with the fact that run-time is also a major constraint of localization attacks, given that a target

TABLE 3: EPs across each numerology in NR.

\begin{tabular}{ccccc}
\hline \hline $\boldsymbol{\mu}$ & \multicolumn{2}{c}{ Standard } & \multicolumn{2}{c}{ CeSAR enhanced } \\
& $\mathrm{EP}=90 \%$ & $\mathrm{EP}=95 \%$ & $\mathrm{EP}=90 \%$ & $\mathrm{EP}=95 \%$ \\
\hline 0 & 150.42 & 190.38 & 121.47 & 150.39 \\
1 & 86.68 & 112.60 & 65.35 & 81.84 \\
2 & 47.41 & 62.93 & 34.17 & 43.08 \\
3 & 25.12 & 34.06 & 17.45 & 22.11 \\
4 & 13.00 & 18.02 & 8.87 & 11.28 \\
\hline
\end{tabular}

${ }^{5} \approx 2.96 \mathrm{~km}$ which is not conducive to mmWave communications could move during computation, we chose 100 runs as our optimal trade-off between accuracy and run-time.

\section{Results}

For the real-world deployment the average MLE error for the applicable numerologies were $30.12 \mathrm{~m}, 12.36 \mathrm{~m}$, and $5.40 \mathrm{~m}$, while for the UD they were $8.64 \mathrm{~m}$ and $4.02 \mathrm{~m}$ respectively. The final location estimates are showcased in Figure 9, where the cyan markers are from the real-world system and the pink markers from the UD system. The cyan markers placement is interesting in that each one is placed nearly linear and equal distances apart starting from the $\mu=0$ marker. We speculate this is entirely due to the the two CeSAR sensors distance estimates' lack of dependence on noise as well as their placement in the environment. The UD errors were in keeping with findings for $\mu=3$ and $\mu=4$ errors in a previous study [21].

\section{Mitigation Techniques}

NR's much lauded increase in physical layer security through massive MIMO, heterogenous networks, tight beamforming, and directionality is highly effective as is for combating the effectiveness of the CeSAR algorithm. CeSAR's necessity to receive the UE's uplink burst in order to estimate distance becomes an extremely challenging, and likely inconsistent, task in 5G networks operating in mmWave bands. Those operating below this, however, without the protection afforded by the extremely thin beams, will still be vulnerable.

Overall this attack is reliant on two portions of the network:

1) TA commands being sent in the clear

2) CRAN fronthaul fiber network

and thus these are the most logical places to mount a defense.

In dealing with the unencrypted TA the only adequate method of security would be to encrypt it. Further efforts at obfuscation, as was the case with the cell-radio network temporary identifier (C-RNTI), do not solve the underlying problem. That being said, encrypting the TA would result in increased latency and increased bandwidth requirements. Increasing latency for timing management could be a nonstarter for many working towards the ultra-low latency communications that $5 \mathrm{G}$ has the potential to offer. To this we suggest a tailored approach, where the end user or a particular application can choose between the added security or the added utility. The CRAN's fiber fronthaul offsets any of the increased physical-layer security measures listed above, as it aggregates the data from potentially thousands of RRHs within a serviceable area to a centralized source, increasing the risk of an adversary collecting data across a much larger area. Without first addressing the issue of encryption, the CRAN architecture as discussed here is a security concern. 


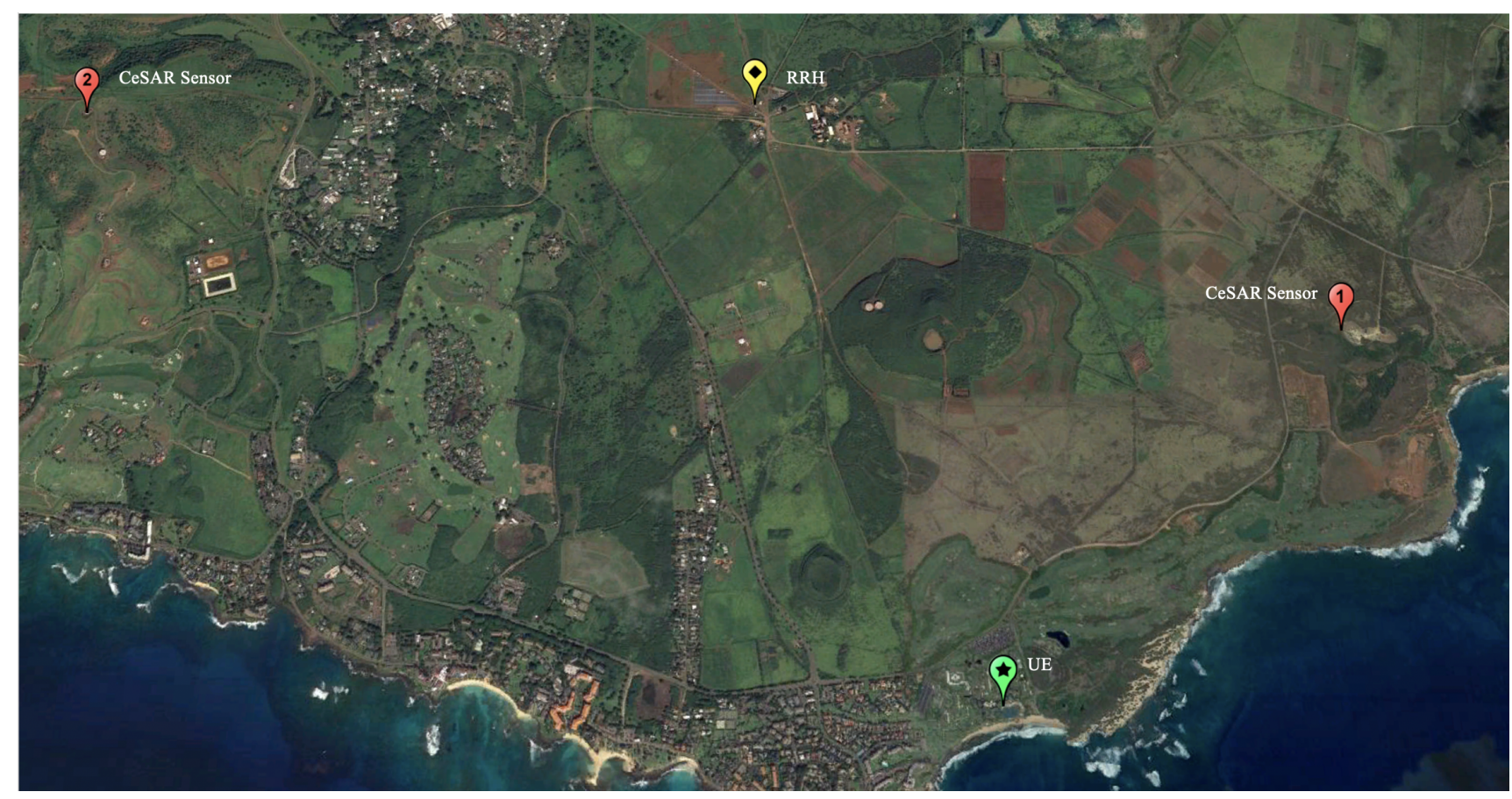

Figure 7: Real-world deployment on Kauai

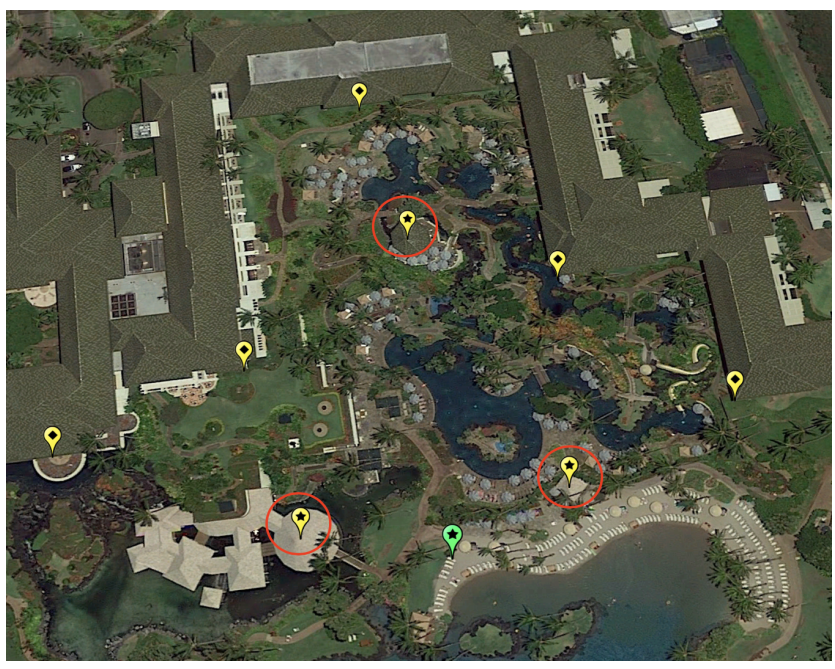

Figure 8: UD deployment on Kauai

\section{Closing Remarks}

We have shown through our investigation that by utilizing aggregated unencrypted TA commands we can calculate statistically efficient location estimates, with some accurate down to $\approx 4$ meters. We have shown that these estimates can be further enhanced using the CeSAR algorithm, and brought to life a vision of what this attack could produce in the real world. However, we have yet to answer our final and most important question posed in the very beginning, "Who cares?" This answer is decidedly split between those who believe that the benefits provided by advances and services

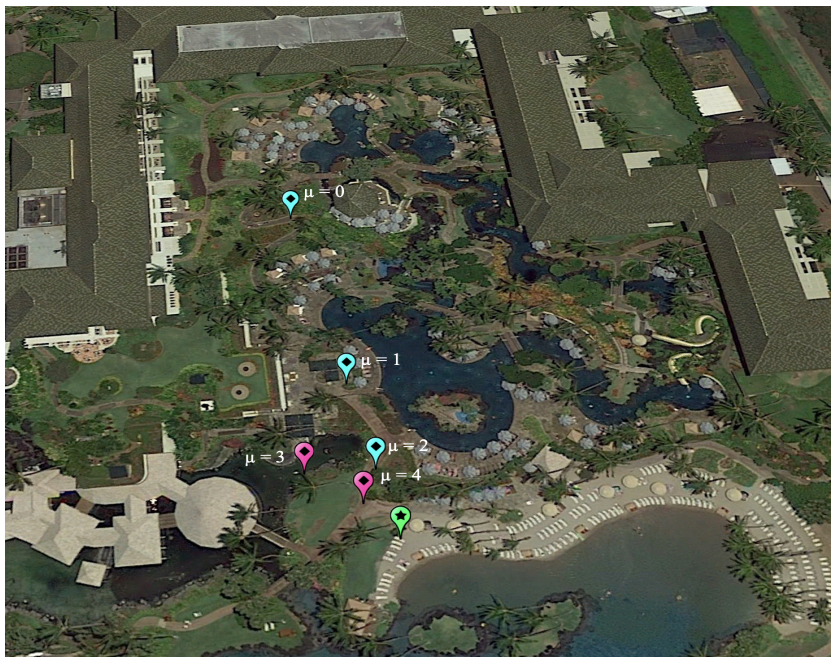

Figure 9: Position estimates for HICSS attendee

are worth the loss in privacy required to make use of them, and those that would do without in order to maintain their sense of privacy. Where these two converge though is around their ability to make a choice, on whether to give up their privacy at all. However, we have shown that regardless of choice, end users' location data is vulnerable. Hearkening back to Professor Westin's definition of privacy, we argue that, as long as this vulnerability exists, we do not have a claim to location privacy. To this we will answer a new question, "Who should care?": everyone. 


\section{List of Acronyms}

$\begin{array}{ll}\text { 5G } & \text { Fifth Generation } \\ \text { SCS } & \text { sub-carrier spacing } \\ \text { LTE } & \text { Long-Term Evolution } \\ \text { CRLB } & \text { Cramer-Rao Lower Bound } \\ \text { CeSAR Cellular Synchronization Assisted Refinement } \\ \text { HICSS } & \text { Hawaii International Conference on System Sci- } \\ & \text { ences } \\ \text { CRAN } & \text { Cloud Radio Access Network } \\ \text { TA } & \text { timing advance } \\ \text { IoT } & \text { Internet of Things } \\ \text { LBS } & \text { location based services } \\ \text { D2D } & \text { device-to-device } \\ \text { UE } & \text { user equipment } \\ \text { FCC } & \text { Federal Communications Commission } \\ \text { E-911 } & \text { enhanced-911 } \\ \text { ToA } & \text { time of arrival } \\ \text { RSS } & \text { received signal strength } \\ \text { AoA } & \text { angle of arrival } \\ \text { RAN } & \text { radio access network } \\ \text { BBU } & \text { baseband unit } \\ \text { RRH } & \text { remote radio head } \\ \text { CPRI } & \text { common public radio interface } \\ \text { MIMO } & \text { multiple-input multiple-output } \\ \text { IEEE } & \text { Institute of Electrical and Electronics Engineers } \\ \text { 3GPP } & \text { 3rd Generation Partnership Project } \\ \text { 5GPP } & \text { 5G Infrastructure Public-Private Partnership } \\ \text { eCPRI } & \text { enhanced CPRI } \\ \text { PLS } & \text { physical layer split } \\ \text { NR } & \text { New Radio } \\ \text { CP } & \text { Cyclic Prefix } \\ \text { TAG } & \text { Timing Advance Group } \\ \text { MLE } & \text { maximum likelihood estimate } \\ \text { C-RNTIcell-radio network temporary identifier } \\ \text { RMSE } & \text { root mean square error } \\ \text { NLLS } & \text { Non-Linear Least Squares } \\ \text { MSE } & \text { mean squared error } \\ \text { CDF } & \text { cumulative distribution function } \\ \text { EP } & \text { circular error probable } \\ \text { RW } & \text { real-world } \\ \text { UD } & \text { ultra-dense } \\ \text { GPS } & \text { global positioning system } \\ \text { 5G } & \text { Fifth Generation } \\ & \end{array}$

\section{References}

[1] A. Westin, Privacy and Freedom. ig Publishing, 1967.

[2] CISCO, "Cisco annual internet report (2018-2023)," CISCO, Tech. Rep., 2020.

[3] Federal Commun. Commission, "Report and order and further notice of proposed rulemaking on revision of the FCC rules to ensure compatibility with enhanced 911 emergency calling systems," pp. 96264, 1996

[4] J. A. del Peral-Rosado, R. Raulefs, J. A. López-Salcedo and G. SecoGranados, "Survey of cellular mobile radio localization methods: From 1G to 5G," in IEEE Communications Surveys Tutorials, 2018, pp. 1124-1148.
[5] FCC, "Fourth report and order on wireless E911 location accuracy requirements," pp. 15-9, Jan. 2015.

[6] S. Perrin, "Evolving to an open c-ran architecture for 5G," Fujitsu, Tech. Rep., 2017.

[7] CPRI Specification V7.0, "Common Public Radio Interface (CPRI); Interface Specification," Oct. 2015.

[8] 3GPP TR 38.801 (V14.0.0), "3rd Generation Partnership Project; Technical Specification Group Radio Access Network; Study on new radio access technology; Radio access architecture and interfaces (Release 14)," Mar. 2017.

[9] eCPRI Specification V1.2, "Common Public Radio Interface; eCPRI Interface Specification,” Jun. 2018.

[10] 3GPP TS 38.213, release 16, (v16.1.0), "3rd Generation Partnership Project; Technical Specification Group Radio Access Network; NR; Physical layer procedures for control," Apr. 2020.

[11] N. Patriciello, S. Lagen, L. Giupponi, and B. Bojovic, "5g new radio numerologies and their impact on the end-to-end latency," in 2018 IEEE 23rd International Workshop on Computer Aided Modeling and Design of Communication Links and Networks (CAMAD), 2018, pp. $1-6$.

[12] 3GPP TS 36.321, release 16, (v16.0.0), "3rd Generation Partnership Project; Technical Specification Group Radio Access Network; Evolved Universal Terrestrial Radio Access (E-UTRA); Medium Access Control (MAC) protocol specification,” Apr. 2020.

[13] E. Dahlman, S. Parkvall, and J. Skold, $5 G N R$ the next generation wireless access technology. Elsevier Ltd., 2018.

[14] 3GPP TS 38.211, release 16, (v16.1.0), "3rd Generation Partnership Project; Technical Specification Group Radio Access Network; NR; Physical channels and modulation," Apr. 2020.

[15] M. DeGroot and M. Schervish, Probability and Statistics, 4th ed. Pearson, 2012.

[16] J. D. Roth, M. Tummala, J. C. McEachen, and J. W. Scrofani, "On location privacy in lte networks," IEEE Transactions on Information Forensics and Security, vol. 12, no. 6, pp. 1358-1368, 2017.

[17] J. D. Roth, M. Tummala, and J. W. Scrofani, "Cellular synchronization assisted refinement (cesar): A method for accurate geolocation in lte-a networks," in 2016 49th Hawaii International Conference on System Sciences (HICSS), 2016, pp. 5842-5850.

[18] I. Guvenc and C. Chong, "A survey on TOA based wireless localization and NLOS mitigation techniques," in IEEE Communications Surveys Tutorials, 2009.

[19] J. J. Caffery and G. L. Stuber, "Overview of radiolocation in CDMA cellular systems," in IEEE Commun. Mag., 1998, pp. 38-45.

[20] D. J. Torrieri, "Statistical theory of passive location systems," in IEEE Trans. Aerosp. Electron. Syst., 1984, pp. 183-197.

[21] A. Schacht, J. Long, and J. Roth, "Timing management in 5g and its implications for location privacy," in 53rd Hawaii International Conference on System Sciences, 2020. 